\title{
QUIZ ON CHILDHOOD EPILEPSIES
}

Q.1 2 month old boy with uneventful birth history started having very frequent focal seizures arising from different regions of the brain. Prolonged VEEG showed migration of the ictal rhythm from one focus to another in some of the prolonged events. MRI brain and metabolic studies were normal. Anomalies in which of the below mentioned genes might lead to this clinical syndrome?
A) KCNT1
B) CDKL5
C) SLC2A1
D) MECP2

Q.2 Which seizure type is the most characteristic of Lennox Gastaut syndrome
A) Atonic seizures
B) Myoclonic seizures
C) Tonic seizures
D) Atypical absence seizures

Q.3 4 year old boy presented with significant global developmental delay with complete absence of speech, facial dysmorphism and myoclonic seizures. His EEG showed the following pattern almost throughout the recording (figure 1).

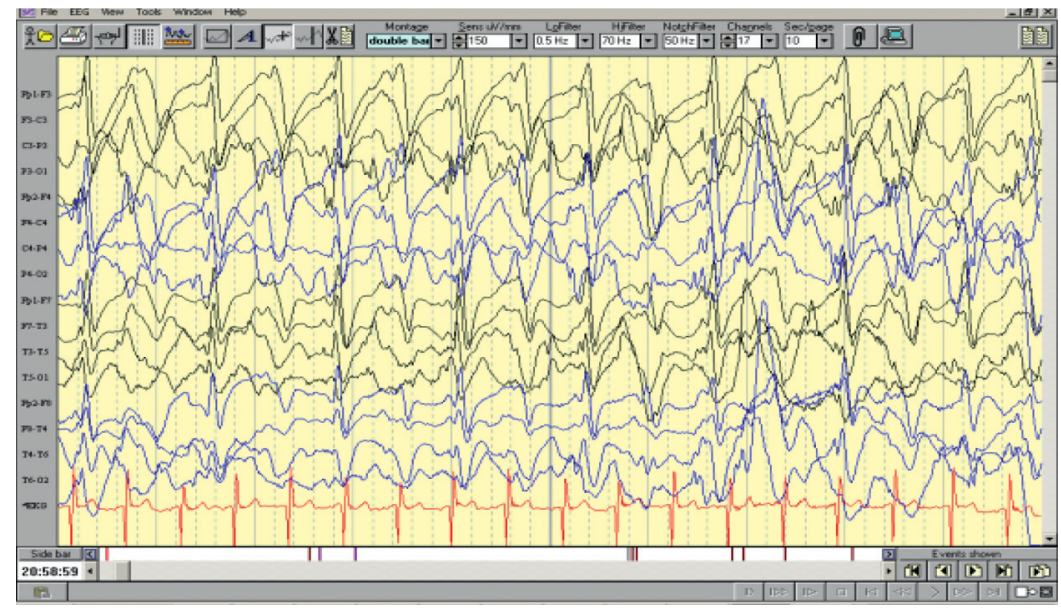

The most likely diagnosis is
A) West syndrome
B) Angelman syndrome
C) Doose syndrome
D) Prader Willi syndrome

Q.4 1 year old boy with uneventful birth history presented with myoclonic seizures and episodes of vacant stare, mostly in the morning hours along with mild developmental delay. His metabolic parameters were normal except for markedly reduced CSF sugar. He was on phenytoin, phenobarbitone, levetiracetam and clobazam. Which one of these, you would like to withdraw
A) Phenytoin
B) Phenobarbitone
C) Levetiracetam
D) Clobazam 
Q.5 One month old baby girl presented with episodes of tonic posturing, cyanosis and frequent myoclonic jerks. Grandmother noticed that almost all of these episodes occurred while the baby was awake and was put in the supine position. The attacks were relieved by flexing the legs. She was started on clonazepam by her pediatrician with significant improvement in the frequency and severity of these events. The most likely diagnosis is
A) Shuddering spells
B) Breath holding spells
C) Jitteriness
D) Hyperekplexia

Q.6 Which one of these newer generation antiepileptic drugs is planned to be withdrawn from the market by the parent company? It is mechanistically related to a childhood epileptic syndrome.
A) Rufinamide
B) Peramapanel
C) Ezogabine
D) Valrocemide

Q.7 6 year old developmentally normal girl presented with recent regression in expressive speech and reading. She had 2 episodes of nonfebrile seizures one year back and was started on an antiepileptic drug. She was completely seizure free afterwards. Her awake and sleep EEGs are given below (figure 2 \& 3 respectively).
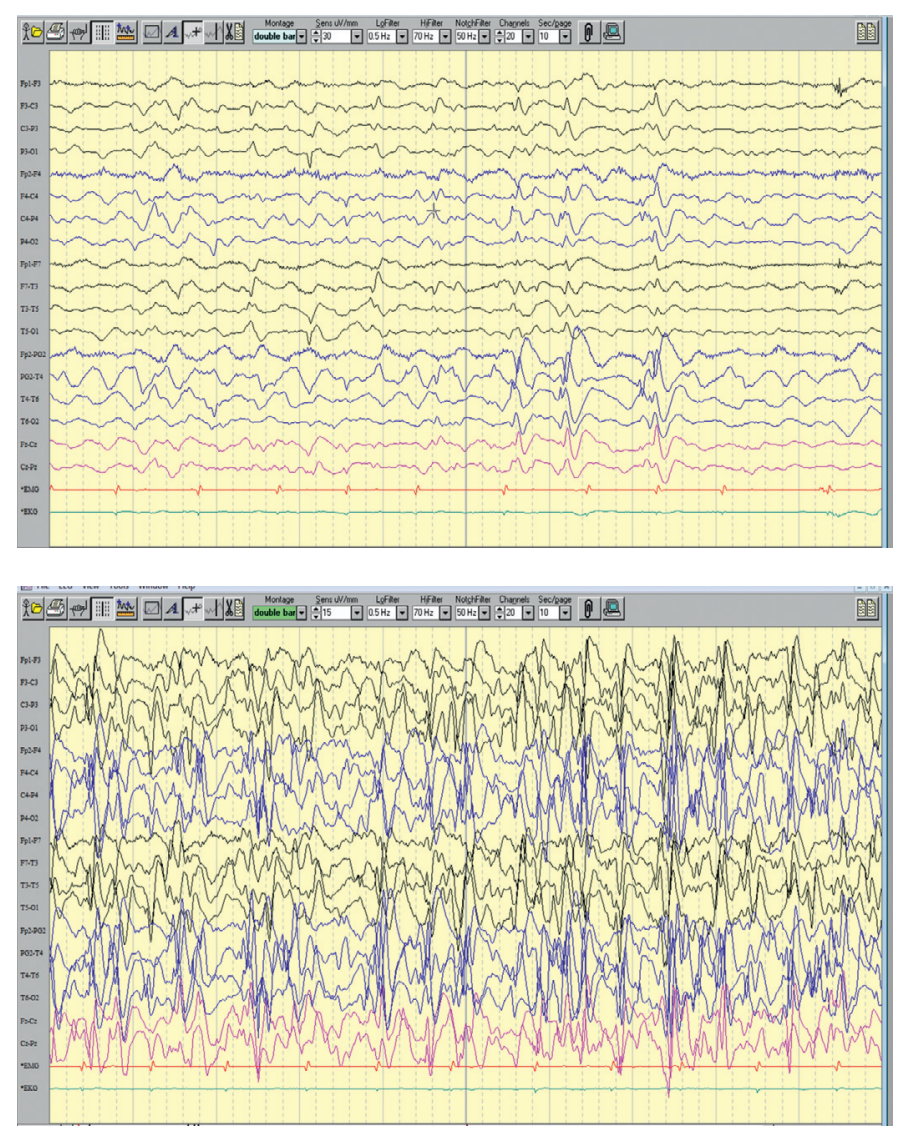

Which one of the following might most probably be the antiepileptic drug she is taking now?
A) Carbamazepine
B) Clobazam
C) Levetiracetam
D) Lamotrigine 
Q.8 NEMO trial failed to demonstrate the efficacy of this drug in neonatal seizures
A) Phenobarbitone
B) Pyridoxine
C) Topiramate
D) Bumetanide

Q.9 This non pharmacologic intervention primarily developed for weight reduction in obese persons has been found to be useful in many drug resistant childhood epilepsies
A) Acupressure
B) Atkins diet
C) Hyperbaric Oxygen
D) Electromagnetic therapy

Q.10 3 month old boy presented with significant floppiness, weakness and seizures. MRI brain showed thick, flat gyri diffusely. Which of the following blood investigations most probably may point towards the diagnosis
A) Elevated lactate
B) High S. CPK
C) Low Hemoglobin
D) High S. Uric Acid

Dr. Vinayan KP MD DM

Professor \& Head Division of Pediatric Neurology Department of Neurology Amrita Institute of Medical Sciences

Cochin, Kerala, India 


\section{Answers}

1. A

Explanation: This clinical syndrome is suggestive of migrating partial epilepsy of infancy, an early infantile epileptic encephalopathy. KCNT1 mutation is the genetic anomaly, most commonly associated with this syndrome. It is also seen in persons with nocturnal frontal lobe epilepsy. MECP2 mutation is usually associated with Rett syndrome. CDKL5 mutation is an X linked disorder mostly seen in girls associated with severe early onset spasms and autistic features similar to Rett syndrome. SLC2 A1 mutations are associated with Glucose transporter deficiency disorder, which usually presents with myoclonic seizures, movement disorders, neurodevelopmental delay or early onset absences.

Ref: On line Mendelian inheritance in Man www.omim.org accessed August 28, 2016

2. C

Explanation: Lennox Gastaut Syndrome (LGS) is an acquired childhood epileptic encephalopathy associated with polymorphic seizures, severe neurodevelopmental delay and generalized slow spike and waves in the EEG. Even though atonic seizures and atypical absences are usually seen in LGS, axial tonic seizures are supposed to be the defining seizure type for this syndrome. Myoclonic seizures are very rare in LGS and the presence of this seizure type would prompt the clinician to consider alternate diagnoses.

Ref: Bureau M, Genton P, Dravet C et al. Epileptic syndromes in Infancy, Childhood and Adolescence (5th ed), John Libbey Eurotext 2012

3. B

Explanation: EEG shows high amplitude frontally dominant "notched" delta pattern which is characteristic of Angelman syndrome. EEG pattern of West syndrome is Hypsarrythmia which is a chaotic mixture of high amplitude spike and slow waves. Doose syndrome may show runs of 4-7 Hz monomorphic theta activity maximum over the centroparietal regions along with generalized polyspikes. Prader Willi syndrome is another genetic imprinting syndrome similar to Angelman syndrome, however with very rare seizures and no characteristic EEG abnormality.

Ref: Bureau M, Genton P, Dravet C et al. Epileptic syndromes in Infancy, Childhood and Adolescence (5th ed), John Libbey Eurotext 2012

Wang PJ, Hou JW, Sue WC, Lee WT. Electroclinical Characteristics of seizures. Comparing Prader Willi syndrome with Angelman Syndrome. Brain Dev. 2005 Mar; 27(2):101-7.

4. B

Explanation: This child is having probable Glucose 1 transporter deficiency. Phenobarbitone is an inhibitor of glucose transporter system and may potentially aggravate hypoglycorrachia and seizures. Ketogenic diet is considered to be the drug of choice in this syndrome

Ref: Wang D, Pascual JM, De Vivo D. Glucose transporter type 1 deficiency syndrome (In) Pagon RA, Adam MP, Ardinger HH, Wallace SE, Amemiya A, Bean LJH, Bird TD, Fong CT, Mefford HC, Smith RJH, Stephens K, editors. GeneReviews ${ }^{\circledR}$ [Internet]. Seattle (WA): University of Washington, Seattle; 1993-2016.

5. D

Explanation: The clinical description strongly favors the possibility of hyperekplexia, a rare autosomal dominant genetic disorder of the glycine receptor characterized by an exaggerated persistent startle reaction to unexpected auditory, somatosensory and visual stimuli, generalized muscular rigidity, and nocturnal myoclonus. Clonazepam, a gamma aminobutyric acid (GABA) receptor agonist, is the treatment of choice for hypertonia and apneic episodes. Vigevano maneuver with forced flexion of the head and legs towards the trunk is known to be life saving when prolonged stiffness impedes respiration in infants.

Ref: Praveen V, Patole SK, Whitehall JS Hyperekplexia in neonates Postgrad Med J 2001;77:570-572 
6. C

Explanation: Retigabine/Ezogabine is a new generation antiepileptic drug which acts as a positive allosteric modulator of potassium channel KCNQ2-5. KCNQ2 mutations are implicated in Benign familial neonatal convulsions and KCNQ2 encephalopathy, a severe infantile epileptic encephalopathy. Retigabine/ Ezogabine was approved by the FDA in 2011 for focal seizures in adults. However, in 2013 FDA warned the public that, Retigabine/Ezogabine can cause blue skin discoloration and eye abnormalities characterized by pigment changes in the retina. In a recent statement, parent manufacturer (GSK) has notified all the stakeholders about their intent to withdraw this drug from the market by June 2017.

Ref: http://www.ilae.org/Visitors/News/documents/GSK_Retigabine_market_withdrawal.pdf accessed Aug 31, 2016

7. A

Explanation: The evolution of symptoms point towards the possibility of Landau-Kleffner syndrome. EEG shows bilateral centrotemporal spike and waves (Right > Left) in awake record (fig 2) along with marked activation in slow wave sleep (CSWS- Fig 3). Carbamazepine is the most commonly implicated antiepileptic drug to induce CSWS in children with benign focal epilepsies, even though the causal association is not very strong. Phenytoin and phenobarbitone are also reported to be associated with this syndrome.

Ref: Nieuwenhuis L, Nicolai J, The pathophysiological mechanisms of cognitive and behavioral disturbances in children with Landau-Kleffner syndrome or epilepsy with continuous spike-and-waves during slow-wave sleep. Seizure 2006; 15, 249-58

8. D

Explanation: Bumetanide is a loop diuretic which is supposed to have a specific role in the management of neonatal seizures as it potentiates the therapeutic effects of phenobarbitone on the immature GABA receptors. However the multicentric Neonatal seizure treatment with Medication Off-patent (NEMO) trial failed to demonstrate the beneficial effect of the combination of Bumetanide with phenobarbitone on neonatal seizure in babies with hypoxic ischemic encephalopathy. This study had to be discontinued prematurely due to the lack of benefit and also due to the unexpected rate of hearing impairment.

Ref: Pressler RM et al. Bumetanide for the treatment of seizures in newborn babies with hypoxic ischaemic encephalopathy (NEMO): an open-label, dose finding, and feasibility phase 1/2 trial. Lancet Neurol 2015; 14: 469-77

9. B

Explanation: The Atkins diet was developed in the United States in 1970 by Robert C. Atkins for the purpose of weight loss. This diet allowed the intake of fat along with the restriction of carbohydrates. The modified Atkins diet is a less restrictive alternative to the traditional ketogenic diet. This diet can be started on an outpatient basis without a fast, allows unlimited protein and fat, and does not restrict calories or fluids. Recent studies have shown good efficacy and tolerability of this diet in refractory childhood epilepsies.

Ref: Sharma S, Sankhyan N, Gulati S, Agarwal A Use of the modified Atkins diet for treatment of refractory childhood epilepsy: a randomized controlled trial. Epilepsia 2013; 54, 481-486

10. B

Explanation: Elevated S. CPK in this clinical setting may point towards the possibility of congenital muscular dystrophy (CMD). Severe brain malformations like lissencephaly may usually be seen in Alpha-dystroglycan associated CMD.

Ref: Bonnemann CG et al . Diagnostic approach to the congenital muscular dystrophies. Neuromuscular Disorders 2014; 24, 289-311 